SYSTEMATIC REVIEW

\title{
The impact of MRI on stroke management and outcomes: a systematic review
}

\author{
James F Burke MD, ${ }^{1}$ Douglas $\mathrm{J} \mathrm{Gelb} \mathrm{MD} \mathrm{PhD,}^{2}$ Douglas J Quint MD, ${ }^{3}$ Lewis B Morgenstern $\mathrm{MD}^{4}$ and \\ Kevin A Kerber MD \\ ${ }^{1}$ Clinical Lecturer, Department of Veterans Affairs, VA Center for Clinical Management and Research, Ann Arbor VA Healthcare System, Robert \\ Wood Johnson Clinical Scholars Program and Department of Neurology, University of Michigan, Ann Arbor, Michigan, USA \\ 2Professor, Department of Neurology, University of Michigan, Ann Arbor, Michigan, USA \\ ${ }^{3}$ Professor, Department of Radiology, University of Michigan, Ann Arbor, Michigan, USA \\ ${ }^{4}$ Professor, Department of Neurology and Department of Epidemiology, School of Public Health, University of Michigan, Ann Arbor, Michigan, USA \\ ${ }^{5}$ Assistant Professor, Department of Neurology, University of Michigan, Ann Arbor, Michigan, USA
}

\author{
Keywords \\ computed tomography, evaluation, magnetic \\ resonance imaging, outcomes, stroke, \\ systematic review \\ Correspondence \\ Dr James F Burke \\ Robert Wood Johnson Clinical Scholars \\ Program \\ University of Michigan Medical School \\ 6312 Medical Science Building 1 \\ Ann Arbor, Ml 48109 \\ USA \\ E-mail: jamesbur@med.umich.edu
}

Disclosures: All authors have seen and agree with the contents of the manuscript. This manuscript is not under review at any other publication. The authors do not have any relevant financial disclosures.

Accepted for publication: 3 October 2012

doi:10.1111/jep.12011

\begin{abstract}
Rationale, aims and objectives Magnetic resonance imaging (MRI) is widely used in stroke evaluation and is superior to computed tomography for the detection of acute ischaemia. We sought to evaluate the evidence that conventional MRI influences doctor management or patient outcomes in routine care.

Methods We systematically searched PubMED, EMBASE and proceedings of the International Stroke Conference. Studies were included if they included patients presenting with possible stroke syndromes and they reported MRI results and resulting changes in management or outcome. Multiple reviewers determined inclusion/exclusion for each study, abstracted study characteristics and assessed study quality.

Results Of 1813 articles screened, nine studies met inclusion criteria. None were randomized controlled trials, cohort studies or case-control studies. We found little evidence that MRI affects outcomes - one single-centre case series presented three patients. The remaining articles were studies of diagnostic tests or vignette-based studies that described changes in doctor management attributed to MRI.

In the studies that suggested MRI influenced management, it did so in two ways. First, MRI distinguished stroke from mimics (e.g. brain tumours), thus enabling more appropriate selection of therapies. Second, even when MRI confirmed a suspected stroke diagnosis, it sometimes provided information (on stroke mechanism, localization, timing or pathophysiology) that influenced management.

Conclusions The impact of MRI on management and outcomes in stroke patients has been inadequately studied. Further research is needed to understand how MRI may productively affect stroke management and outcomes.
\end{abstract}

\section{Introduction}

Stroke is common [1] and is responsible for substantial disability $[2,3]$ and costs [4]. The use of magnetic resonance imaging (MRI) in the diagnosis and management of stroke has substantially increased over the last decade [5].

Stroke patients typically require a neuroimaging study, either computed tomography (CT) or MRI, to differentiate ischaemic stroke from intracerebral haemorrhage [6]. When the diagnosis of stroke is uncertain, MRI is the more informative of the two imaging modalities [7-11]. When the diagnosis of stroke is reasonably certain, however, the value of MRI (in addition to or instead of CT) is less clear. In principle, MRI-derived information about stroke mechanism [12-14], localization [15,16], timing [17-20] and pathophysiology [21] could improve decisions regarding acute management and secondary prevention even when the diagnosis is clear cut. This may be why in some parts of the country, MRI is obtained on nearly all stroke patients (except those with MRI contraindications) [5]. Utilization of MRI varies widely between states however [5]. Given the established superiority of MRI in cases where the diagnosis is uncertain, at least some of this interstate variability probably reflects a lack of consensus regarding the value of MRI in cases where the diagnosis of stroke can be established clinically. Empiric evidence bearing on this issue could help to standardize clinical practice and make it more costeffective. To address this question, we performed a systematic 
review of the evidence that stroke patients evaluated with MRI are managed differently or have different outcomes than patients evaluated with other imaging strategies.

\section{Methods}

We sought to identify all published studies documenting that conventional MRI-based structural brain imaging led to changes in patient management or outcomes, and to classify those changes. We adhered to a pre-specified protocol detailing our search strategy, inclusion/exclusion criteria, procedures for determining inclusion/exclusion and quality assessment methodology using the PRISMA guidelines for systematic reviews [22].

\section{Study eligibility for inclusion}

To be included in our review, a study had to involve patients over the age of 18 who presented with acute stroke-like symptoms; some subset of the patients underwent MRI imaging; and structural MRI results were reported in the paper. In addition, studies had to either (1) describe a change in patient management that would not have occurred had MRI not been performed or (2) describe a change in patient outcomes in patients who underwent MRI compared to patients who did not.

We included all studies regardless of study design with the exception of case reports. Consequently, we included both studies directly comparing MRI to CT as well as other studies comparing one MRI technique to another. While studies comparing MRI techniques do not enable direct inferences about the role of MRI versus $\mathrm{CT}$, we concluded that such inferences were reasonable. If, for example, a study showed that MRI with diffusion-weighted imaging (DWI) changed management compared to MRI without, it is reasonable to infer that MRI with DWI would change management compared to CT. We excluded studies that only enrolled patients with transient ischaemic attack. We also excluded studies where management changes were based on multi-modal MRI (e.g. MRA, MR perfusion) as opposed to structural MRI of the brain parenchyma, for two reasons. First, we wanted to focus on the type of information that can only be obtained with MRI - angiographic and perfusion imaging information can also be obtained using CT-based technology and conventional angiography. Second, we wanted to focus on the way MRI is most widely used in the community; multi-modal MRI has limited availability in the emergency department [23].

\section{Information sources and search}

We worked with an experienced research librarian to develop comprehensive search strategies for studies combining three major themes: stroke, MRI and changes in management or outcomes (search strings available as Supporting Information Appendix S1). PubMED and EMBASE were separately searched. All studies available in English were included, regardless of publication date, through 2 December 2010 (the date our search was performed). In addition, we hand searched all published abstracts of the proceedings of the International Stroke Conference (ISC) from 2001 to 2010 for other studies that might meet inclusion criteria. When relevant abstracts were identified, full-length articles were sought by serially searching on the first author's name, the last author's name and pertinent keywords [24].

\section{Study selection}

After performing our search, one reviewer (JB) screened the PubMED/EMBASE search results and ISC proceedings to identify candidate abstracts, and then evaluated those abstracts to identify articles that potentially met our inclusion/exclusion criteria. At both stages, an article was rejected only if it clearly failed to satisfy inclusion criteria or clearly met exclusion criteria. Next, two authors reviewed the full text of each of the 74 non-rejected articles to arrive at final inclusion/exclusion determinations. Agreement between reviewers was good (kappa $=0.66,95 \% \mathrm{CI}$ $0.42-0.90)$. Disagreements were resolved by group consensus. We categorized the nine articles that were ultimately determined to meet our criteria into one of two groups, depending on whether the article described changes in management or changes in outcomes (Fig. 1).

\section{Data extraction}

In order to identify the features relevant to quality assessment, two reviewers independently evaluated each included study and recorded the primary research question, study design, number of subjects included relevant to our study question, whether data was retrospectively or prospectively acquired and the target population. The primary summary measures were the number of patients in whom management or outcome were changed. Differences were resolved by consensus.

\section{Quality assessment}

We analysed each included study using a modified version of the QUADAS tool [25]. QUADAS consists of 14 yes/no questions that address the key components of quality in studies of diagnostic tests. This tool has primarily been used to measure the quality of studies of diagnostic accuracy, so we added three additional questions to capture the nature of changes in management and outcomes in these studies.

Two reviewers evaluated each study on each QUADAS question. Overall agreement was moderate (kappa $=0.48,95 \% \mathrm{CI}$ 0.35-0.61). Differences were resolved by consensus.

\section{Results}

A total of 1813 articles were identified by the search strategy. Of these, 424 were selected for abstract review and 74 for full-text review. Ultimately, nine articles were determined to meet full inclusion/exclusion criteria (Fig. 1).

All nine of these articles described single-centre studies. They incorporated a variety of study designs, targeted different patient populations and compared different imaging strategies (e.g. CT versus MRI; MRI with DWI versus MRI without DWI) [26-34]. In light of this heterogeneity, we reported study results narratively and did not attempt to pool study results. Only one study, a 


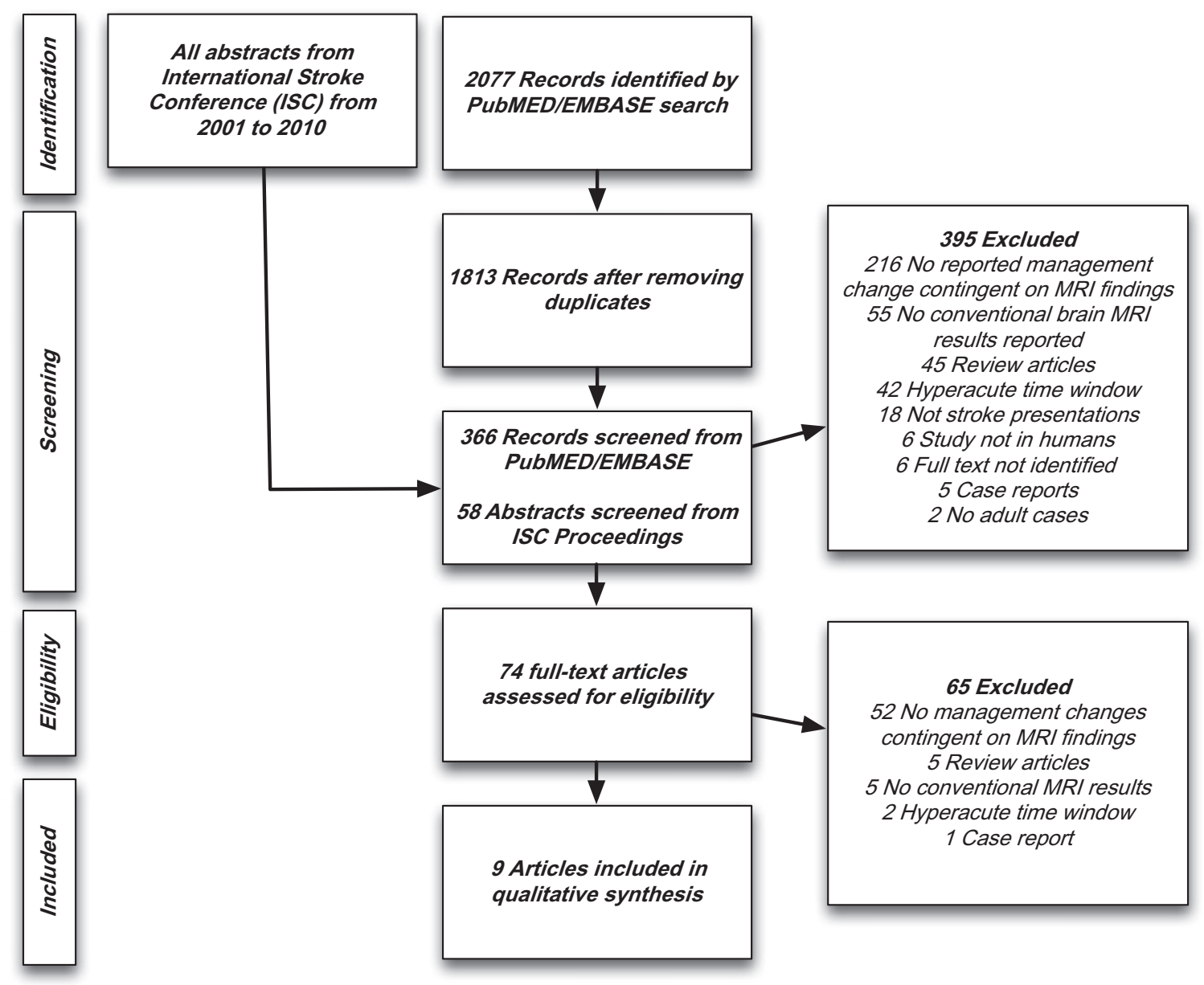

Figure 1 Summary of inclusion/exclusion strategy.

three-patient case series, described changes in outcomes attributed to MRI findings [31]. The remaining eight studies documented instances in which MRI findings resulted in changes in doctor management, but did not demonstrate that these changes in management affected outcomes. Further details of the included studies are summarized in Table 1 and Supporting Information Appendix S2. Figure 2 summarizes the ways in which MRI findings altered clinical assessment and management, separated into two broad categories: (1) situations in which MRI led to diagnostic revision (i.e. patients whose post-MRI diagnosis differed from their pre-MRI diagnosis); or (2) situations in which the pre-MRI diagnosis and post-diagnosis MRI were both stroke, but the MRI provided additional information that affected management. Supporting Information Appendix S3 summarizes study quality.

\section{Impact of MRI}

\section{Changes in outcomes}

We identified only one study, the small case series from WaldronLynch et al. [31], that described changes in outcome attributable to MRI: three patients with presumed posterior circulation strokes had their diagnoses changed to posterior reversible encephalopathy syndrome (PRES), leading to changes in blood pressure management and favourable outcomes.

\section{Changes in management}

\section{Change in management due to change in diagnosis}

Diagnostic revision attributed to MRI was described in five studies, four of which documented changes in management as a consequence of the revised diagnoses. In the study of Kreisl et al. [32], MRI evidence of stroke influenced the decision to initiate or change secondary stroke prevention strategies in 14 of 66 patients with primary brain tumours. In the series of 116 patients reported by Shuaib et al. [26], of the 22 patients in whom MRI changed management, the change was due to revised diagnosis in two cases. One patient with suspected stroke was started on anticoagulation when MRI revealed unsuspected dural venous thrombosis. Another patient was suspected to have dural venous thrombosis prior to MRI, and a planned angiogram was averted when the MRI showed no thrombosis. In four patients with normal MRIs presenting within 3 hours of onset, Sunshine et al. [33] withheld planned intravenous (IV) thrombolysis when no infarct was visualized on MRI. 


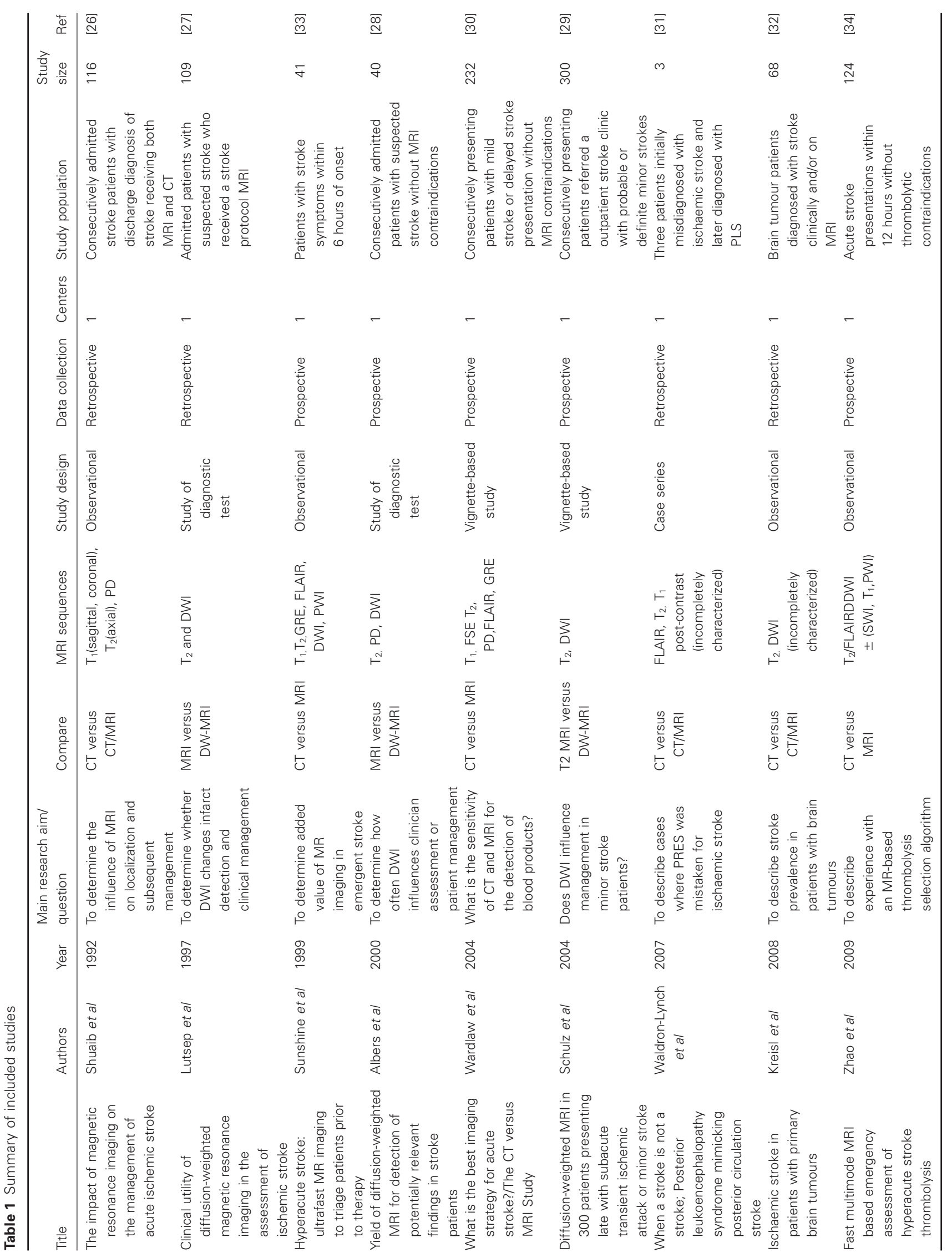




\section{Change thinking}

\section{Change management}

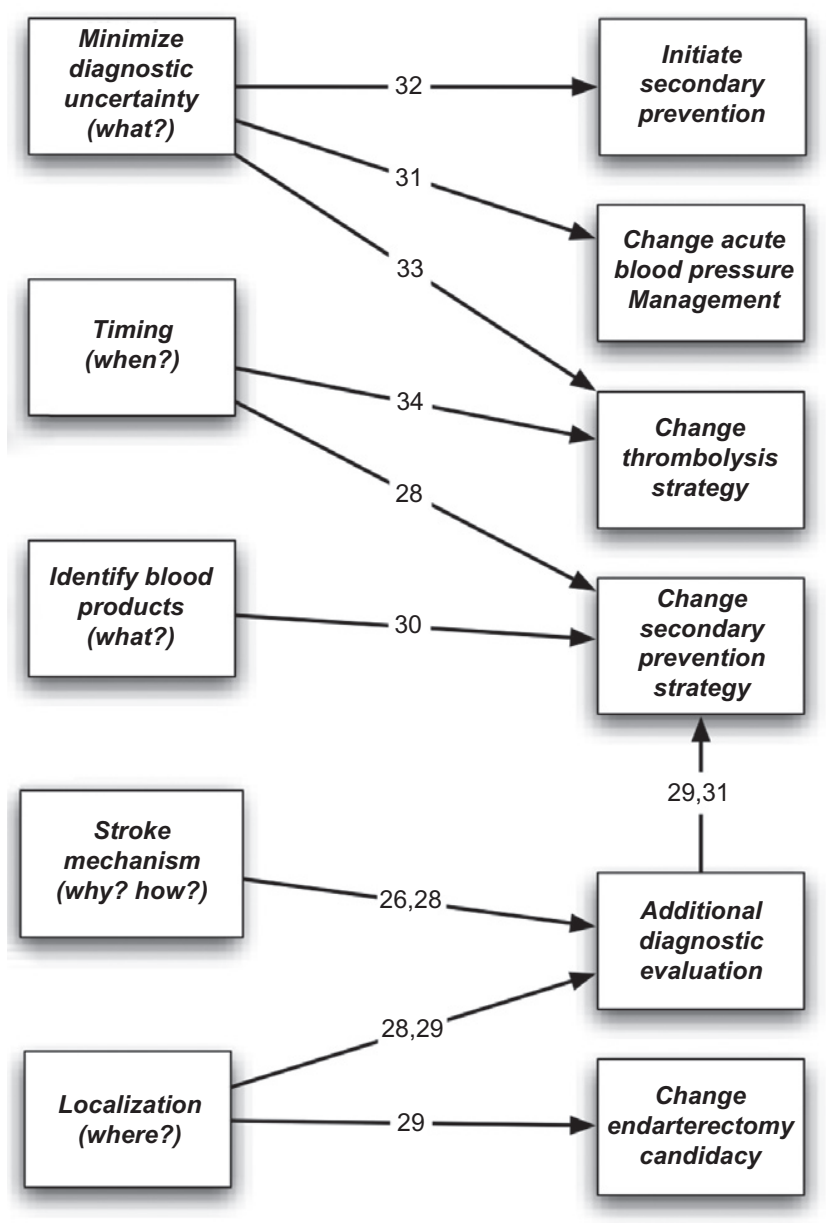

Figure 2 Summary of management changes identified in included articles.

Change in management due to additional information without change in diagnosis

Even in cases where the pre-MRI diagnosis and post-MRI diagnosis were both stroke, MRI sometimes revealed information that led to changes in management. In some cases, the additional information concerned stroke location (and consequently, stroke mechanism) [27-29]. In other cases, the MRI provided direct information regarding stroke mechanism, by differentiating haemorrhage from ischaemia or by demonstrating thrombus directly $[26,30]$. In two other cases, the MRI permitted inferences regarding stroke timing [28,34].

Three studies reported patients who were managed differently because of the information about stroke location derived from MRI. Schulz et al. [29] found that DWI findings changed clinicians' conclusions regarding anterior versus posterior circulation in 27 of $164(16 \%)$ patients with mild stroke. Carotid endarterectomy was performed in four of the patients for whom DWI results changed the presumed stroke location from the posterior to the anterior circulation, and carotid imaging was not obtained in 10 patients after DWI changed the presumed stroke location from the anterior to the posterior circulation. Lutsep et al. [27] described one patient out of 109 with a presumed anterior circulation stroke that was relocalized to the pons after MRI, leading to vascular imaging, which revealed basilar stenosis, prompting a decision to recommend anticoagulation. Albers et al. [28] described a single patient in their series of 40 who was initially suspected to have a lacunar infarct until MRI revealed a cortical localization, inducing the treating doctors to order a transthoracic echocardiogram (TTE) that would not have been obtained if MRI had confirmed a lacunar infarct. The TTE demonstrated a large patent foramen ovale and the patient was placed on anticoagulation because of a clinical suspicion of paradoxical embolus.

Improved stroke localization was not the only way in which MRI provided information regarding stroke mechanism. Shuaib et al. [26] identified one patient whose MRI detected an unanticipated acute carotid thrombus, leading the treating doctors to initiate anticoagulation. Wardlaw et al. [30] found that MRI identified both primary intracerebral haemorrhage and recent infarct with haemorrhagic transformation more commonly than $\mathrm{CT}$, resulting in altered antithrombotic management in 14 out of the 232 enrolled patients, including five patients where MRI identified ICH that was not detected on CT.

The superiority of MRI over CT, with respect to determining the age of an infarct, led to changes in management in at least one of the patients reported by Albers et al. [28]. That patient presented with new focal neurologic symptoms initially thought to be the consequence of a new infarct, but subsequently classified as unmasking of old stroke symptoms based on MRI results. Consequently, the patient was continued on warfarin monotherapy (for known atrial fibrillation) instead of starting an additional antiplatelet agent as was planned at the time of initial clinical assessment. Zhao et al. [34] also used MRI to clarify the age of infarcts. By treating all patients with hyperacute MRI findings who presented within 12 hours from onset, 39 patients who would not have received IV thrombolysis based on a conventional time-based treatment strategy received thrombolysis.

\section{Study quality}

We evaluated study quality using the QUADAS instrument (Supporting Information Appendix S3). With respect to this standard, quality varied considerably between studies. The two most common methodological limitations were inclusion of the index test in the reference diagnostic standard and failure to report withdrawals from the study. Many of the examples of management changes cited in the studies were inconsistent with the most recent evidence-based guidelines [35,36], but this is not surprising given that most of the studies were published more than 5 years ago.

\section{Discussion}

This systematic review identified almost no direct evidence that MRI affects outcome in patients with stroke, and limited evidence - derived from a small number of studies - that MRI affects management. Of the 1813 screened articles, only nine explicitly discussed how MRI findings influence management and only one of these (a small case series) provided evidence suggesting that 
MRI findings contribute to improved patient outcomes. All identified studies were single-centre series; we found no randomized controlled trials (RCTs), cohort studies or case-control studies that addressed our specific study question. Many of the examples of management changes cited in the nine studies were inconsistent with the most recent evidence-based guidelines [35,36]. This modest evidence base does not justify the routine use of MRI in situations where the diagnosis of stroke is unequivocal.

Even though we did not find evidence from strong study designs that MRI findings translate into changes in management or improved outcomes, the studies included in our review illustrate possible pathways linking MRI to outcomes. Specifically, they provide examples in which MRI information about diagnosis, stroke location or timing led clinicians to change management. These suggestive examples merit more extensive and rigorous investigation. If confirmed, they could point the way to more effective stroke management algorithms and improved patient selection for MRI. Given the high prevalence of stroke and the cost of MRI, the clinical and public policy implications could be substantial.

The value of this additional MRI-derived information cannot be determined simply by evaluating sensitivities and specificities without also studying how the additional information translates into changes in management. The ideal would be to perform RCTs, but these studies may not be practical for stroke MRI given that MRI is now considered routine by many clinicians and given the cost required to follow large number of stroke patients over an extended interval.

Some of the studies included in our review provide examples of study designs that might serve as alternatives to RCTs. In particular, the vignette-based approaches of Wardlaw et al. and Schulz et al. illustrate the advantages of requiring clinicians to state how they would manage patients based on real-world cases, first without and then with knowledge of MRI results. With this study design, patients serve as their own controls, the exposure of interest can be precisely controlled, and the use of consecutive real-world cases allows for estimates of how frequently management is changed in practice. Limitations include the artificial nature of the case abstraction and the decision-making environment. Some of these limitations can be circumvented using a study design similar to that of Albers et al. [28], requiring the treating clinicians to commit to an explicit diagnostic impression and management plan immediately before and immediately after obtaining an MRI. Neither vignettebased studies nor pre-MRI/post-MRI surveys of treating doctors can directly assess clinical outcomes, but they could provide the background information necessary to perform simulation studies.

Our review had several limitations. First, although our search criteria were designed to be as broad as possible, they may have failed to identify some articles that would meet our inclusion criteria. Second, we only included studies that provided explicit examples of how MRI information altered management. Thus, studies were excluded if management changes were only implicit. Third, study methodology varied widely. Most notably, some included studies compared MRI to CT and others compared MRI techniques. Finally, we can not draw any conclusions regarding the role of multi-modal MRI [e.g. magnetic resonance angiography (MRA) or MR perfusion] because we included only studies in which the management changes were based on structural MRI of the brain parenchyma.
These limitations actually reinforce our conclusion that the available evidence is insufficient to determine the appropriate role of stroke MRI. Its use is justified when the diagnosis of stroke is unclear [11], but the limited evidence base linking MRI to changes in management or outcomes calls into question the routine use of stroke MRI in all stroke patients. Of course, the absence of evidence relating MRI to outcomes is not evidence of no effect. The nine studies identified in this review do tend to support the common impression that MRI provides information (not available from CT) that influences management in some stroke scenarios. Unfortunately, these studies do not justify general inferences regarding how often this is the case, or in what circumstances. Despite the substantial methodological difficulties inherent in investigating these questions more rigorously, the clinical and policy implications are great enough to justify the effort that will be required.

\section{Acknowledgements}

The authors are grateful for the significant assistance and support provided by Marisa Conte, Clinical and Translational Science liaison, at the University of Michigan Taubman Health Sciences Library.

JFB is supported by the Robert Wood Johnson Foundation Clinical Scholars Program and an associated Veterans Affairs (VA) Advanced Fellowship.

KAK received research support from NIH grant K23 RR024009 and AHRQ grant R18 HS017690, and received honorarium for invited presentations on topics related to dizziness for the American Academy of Neurology 2010 Annual Meeting.

LBM received research support from NIH grant R01NS038916 and AHRQ grant R18 HS017690. Research support from St. Jude Medical Corp.

Medicolegal work (not for industry) and less than 10k per year.

Grant support from NIH: R01NS062675, R01NS038916, U01NS062835, U54NS057405, U01NS056975, U01NS052510 and AHRQ R18HS017690.

DJG and DJQ have nothing to disclose

\section{References}

1. Roger, V. L., Go, A. S., Lloyd-Jones, D. M., Adams, R. J., Berry, J. D., Brown, T. M., et al. (2010) Heart disease and stroke statistics - 2011 update: a report from the American Heart Association. Circulation, 123, e18-e209.

2. Centers for Disease Control and Prevention (2009) Prevalence and most common causes of disability among adults. MMWR. Morbidity and Mortality Weekly Report, 58 (16), 421-426.

3. Adamson, J., Beswick, A. \& Ebrahim, S. (2004) Is stroke the most common cause of disability? Journal of Stroke and Cerebrovascular Diseases, 13 (4), 171-177.

4. Brown, D. L., Boden-Albala, B., Langa, K. M., Lisabeth, L. D., Fair, M., Smith, M. A., et al. (2006) Projected costs of ischemic stroke in the United States. Neurology, 67 (8), 1390-1395.

5. Burke, J. F., Kerber, K. A., Iwashyna, T. J. \& Morgenstern, L. B. (2012) Wide variation and rising utilization of stroke magnetic resonance imaging: data from 11 states. Annals of Neurology, 71 (2), $179-185$.

6. Goldstein, L. \& Simel, D. (2005) Is this patient having a stroke? JAMA: the Journal of the American Medical Association, 293 (19), 2391-2402. 
7. Barber, P. A., Hill, M. D., Eliasziw, M., Demchuk, A. M., Pexman, J. H. W., Hudon, M. E., et al. (2005) Imaging of the brain in acute ischaemic stroke: comparison of computed tomography and magnetic resonance diffusion-weighted imaging. Journal of Neurology, Neurosurgery, and Psychiatry, 76 (11), 1528-1533.

8. Chalela, J., Kidwell, C., Nentwich, L. \& Luby, M. (2007) Magnetic resonance imaging and computed tomography in emergency assessment of patients with suspected acute stroke: a prospective comparison. Lancet, 369, 293-298.

9. González, R. G., Schaefer, P. W., Buonanno, F. S., Schwamm, L. H., Budzik, R. F., Rordorf, G., et al. (1999) Diffusion-weighted MR imaging: diagnostic accuracy in patients imaged within 6 hours of stroke symptom onset. Radiology, 210 (1), 155-162.

10. Fiebach, J. B., Schellinger, P. D., Jansen, O., Meyer, M., Wilde, P., Bender, J., et al. (2002) CT and diffusion-weighted MR imaging in randomized order: diffusion-weighted imaging results in higher accuracy and lower interrater variability in the diagnosis of hyperacute ischemic stroke. Stroke, 33 (9), 2206-2210.

11. Brazzelli, M., Sandercock, P. A., Chappell, F. M., Celani, M. G., Righetti, E., Arestis, N., et al. (2009) Magnetic resonance imaging versus computed tomography for detection of acute vascular lesions in patients presenting with stroke symptoms. Cochrane Database of Systematic Reviews, (4)CD007424.

12. Barber, P. A. (2006) Is diffusion-weighted imaging helpful in determining the source of stroke? Commentary. Nature Reviews Neurology, 2, 424-425.

13. Gass, A., Ay, H., Szabo, K. \& Koroshetz, W. J. (2004) Diffusionweighted MRI for the 'small stuff': the details of acute cerebral ischaemia. Lancet Neurology, 3 (1), 39-45.

14. Wessels, T., Wessels, C., Ellsiepen, A., Reuter, I., Trittmacher, S., Stolz, E., et al. (2006) Contribution of diffusion-weighted imaging in determination of stroke etiology. AJNR. American Journal of Neuroradiology, 27 (1), 35-39.

15. Flossmann, E., Redgrave, J. N., Briley, D. \& Rothwell, P. M. (2008) Reliability of clinical diagnosis of the symptomatic vascular territory in patients with recent transient ischemic attack or minor stroke. Stroke, 39 (9), 2457-2460.

16. Lansberg, M., Albers, G., Beaulieu, C. \& Marks, M. (2000) Comparison of diffusion-weighted MRI and CT in acute stroke. Neurology, 54 (8), 1557-1561.

17. Ebinger, M., Galinovic, I., Rozanski, M., Brunecker, P., Endres, M. \& Fiebach, J. B. (2010) Fluid-attenuated inversion recovery evolution within 12 hours from stroke onset: a reliable tissue clock? Stroke, 41 (2), 250-255.

18. Aoki, J., Kimura, K., Iguchi, Y., Shibazaki, K., Sakai, K. \& Iwanaga, T. (2010) FLAIR can estimate the onset time in acute ischemic stroke patients. Journal of the Neurological Sciences, 293 (1-2), 39-44.

19. Axer, H., Grässel, D., Brämer, D., Fitzek, S., Kaiser, W. A., Witte, O. W., et al. (2007) Time course of diffusion imaging in acute brainstem infarcts. Journal of Magnetic Resonance Imaging, 26 (4), 905-912.

20. Fiebach, J. B., Jansen, O., Schellinger, P. D., Heiland, S., Hacke, W. \& Sartor, K. (2002) Serial analysis of the apparent diffusion coefficient time course in human stroke. Neuroradiology, 44 (4), 294-298.

21. Bryan, R., Levy, L., Whitlow, W., Killian, J., Preziosi, T. \& Rosario, J. (1991) Diagnosis of acute cerebral infarction: comparison of CT and MR imaging. AJNR. American Journal of Neuroradiology, 12 (4), 611-620.

22. Moher, D., Liberati, A., Tetzlaff, J., Altman, D. G. \& Group, P. (2009) Preferred reporting items for systematic reviews and meta-analyses: the PRISMA statement. Annals of Internal Medicine, [Guideline]. 151 (4), 264-269.

23. Centers for Disease Control and Prevention National hospital ambulatory medical care survey: 2008 emergency department summary tables (table 17). Atlanta. Available at: http://www.cdc.gov/nchs/data/ ahcd/nhamcs_emergency/nhamcsed2008.pdf (last accessed 9 September 2011)

24. Sanossian, N., Ohanian, A. G., Saver, J. L., Kim, L. I. \& Ovbiagele, B. (2006) Frequency and determinants of nonpublication of research in the stroke literature. Stroke, 37 (10), 2588-2592.

25. Whiting, P., Rutjes, A. W. S., Reitsma, J. B., Bossuyt, P. M. M. \& Kleijnen, J. (2003) The development of QUADAS: a tool for the quality assessment of studies of diagnostic accuracy included in systematic reviews. BMC Medical Research Methodology, [Evaluation Study]. 3, 25.

26. Shuaib, A., Lee, D., Pelz, D., Fox, A. \& Hachinski, V. (1992) The impact of magnetic resonance imaging on the management of acute ischemic stroke. Neurology, 42, 816-818.

27. Lutsep, H. L., Albers, G. W., DeCrespigny, A., Kamat, G. N., Marks, M. P. \& Moseley, M. E. (1997) Clinical utility of diffusion-weighted magnetic resonance imaging in the assessment of ischemic stroke. Annals of Neurology, 41 (5), 574-580.

28. Albers, G. W., Lansberg, M. G., Norbash, A. M., Tong, D. C., O’Brien, M. W., Woolfenden, A. R., et al. (2000) Yield of diffusion-weighted MRI for detection of potentially relevant findings in stroke patients. Neurology, 54 (8), 1562-1567.

29. Schulz, U. G., Briley, D., Meagher, T., Molyneux, A. \& Rothwell, P. M. (2004) Diffusion-weighted MRI in 300 patients presenting late with subacute transient ischemic attack or minor stroke. Stroke, 35 (11), 2459-2465.

30. Wardlaw, J. M., Keir, S. L., Seymour, J., Lewis, S., Sandercock, P. A. G., Dennis, M. S., et al. (2004) What is the best imaging strategy for acute stroke? Health Technology Assessment, 8 (1), iii, ix-x, 1-180.

31. Waldron-Lynch, F., Meehan, C., Roche, C., Murphy, J., Hennessy, M. \& Counihan, T. J. (2007) When a stroke is not a stroke; posterior leukoencephalopathy syndrome mimicking posterior circulation stroke. Irish Medical Journal, 100 (1), 334-336.

32. Kreisl, T. N., Toothaker, T., Karimi, S. \& DeAngelis, L. M. (2008) Ischemic stroke in patients with primary brain tumors. Neurology, 70 (24), 2314-2320.

33. Sunshine, J., Tarr, R., Lanzieri, C. \& Landis, D. (1999) Hyperacute stroke: ultrafast MR imaging to triage patients prior to therapy1. Radiology, 212 (2), 325-332.

34. Zhao, Z., Bai, Q., Sui, H., Xie, X. \& Wen, F. (2009) Fast multimode MRI based emergency assessment of hyperacute stroke thrombolysis. Neurological Research, 31 (4), 346-350.

35. Adams, J. H. P., Del Zoppo, G. J., Alberts, M. J., Bhatt, D. L., Brass, L. M., Furlan, A., et al. (2007) Guidelines for the early management of adults with ischemic stroke: a guideline from the American Heart Association/American Stroke Association. Stroke, 38 (5), 1655-1711.

36. Furie, K. L., Kasner, S. E., Adams, R. J., Albers, G. W., Bush, R. L., Fagan, S. C., et al. (2011) Guidelines for the prevention of stroke in patients with stroke or transient ischemic attack: a guideline for healthcare professionals from the American Heart Association/American Stroke Association. Stroke, 42 (1), 227-276.

\section{Supporting information}

Additional Supporting Information may be found in the online version of this article:

Appendix S1 Search strings. Appendix S2 Summary of studies.

Appendix S3 Results of QUADAS quality evaluation.Columns represent QUADAS questions and rows represent individual studies. 